\title{
Analysis on the Design Elements and Strategies of Horticultural Therapy in the Healing Environment of Patients with Limb Dysfunction*
}

\author{
Jing Guo \\ School of Design \\ Southwest Forestry University \\ Kunming, China 650224
}

\author{
Zhao $\mathrm{Xu}^{* *}$ \\ School of Design \\ Southwest Forestry University \\ Kunming, China 650224 \\ **Corresponding Author
}

\begin{abstract}
According to the healing and treatment of patients with limb dysfunction, the psychological features and requirements for healing of patients with limb dysfunction have been analyzed in this paper. The environment, plants and patients of implementing horticultural therapy for patients with limb dysfunction are taken as the design elements to explore the strategies of the safe and accessible place, the clear target of plant furnishing, establishing multiple experience space and sound treatment plans and management methods for the horticultural therapy, in order to create a healing space for patients with limb dysfunction and promote the diversified development of healing environment design.
\end{abstract}

Keywords-patients with limb dysfunction; healing environment; horticultural therapy; design elements; methods and strategies

\section{INTRODUCTION}

A series of significant decisions and arrangements such as "implementing the strategy of health in China" have been specified in the report of the 19th National Congress of the Communist Party of China. It puts forward "People's health is an important symbol of national prosperity and strength". It symbolizes the health industry has entered the period with booming development. The national health project is complicated and systematic and needs to integrate multiple disciplines such as medicine, pharmacy, environics, botany and rehabilitation science, so that it will truly guarantee human health. Horticultural therapy refers to a new type interdisciplinary science that integrates medicine, pharmacy, environics, botany, ecology, physiology, psychology, rehabilitation science, sociology and ergonomics. As a relatively mature method and strategy in the fields of modern medical health and environmental construction, it will effectively promote people's physical and psychological health, spiritual pleasure and long life and has achieved sound healing efficacy in the fields such as rehabilitation care, spiritual care, disabled people, mentally retarded children, special education and nursing care of the elderly.

*Fund program: The phased achievement of youth found project for humanities and social science research of the Ministry of Education (Project No.: 15YJC760033)

\section{INTERPRETING THE DEFINITION OF HORTICULTURAL THERAPY}

The horticultural therapy originated from Scotland at the early 18th century. In the 1970s, America founded the world's first Horticultural Therapy Association that has developed for decades of years. An increasing number of countries have involved in the industry of horticultural therapy.

The horticultural therapy is an occupational therapy. According to the characteristics of patients' symptom, it purposefully chooses and arranges activities such as plant cultivation and horticultural operation in the healing environment, in order to adjust and promote patients' somatic function and the healing and treatment effect of mental disease. The information of American Horticultural Therapy Association shows horticultural therapy refers to an effective method to adjust and update physiological and mental diseases. Japanese Horticultural Therapy Seminar thinks the horticultural therapy can flexibly apply the good effect produced by horticultural operation and is a medical method of healing without side effect to aid and treat human diseases. On this basis, the horticultural therapy is defined as an adjuvant therapy to heal patients' somatic function and mental function according to the process that people participate in the horticultural operation. In this process, people have closer contact and interaction with various activities related to the growth of plants. It is evident that the horticultural therapy is a medical method of healing that integrates light manual labor and light brain work. It mainly relieves the mental stress of patients, promotes blood circulation and lowers blood pressure as well as protects joints through adjusting central nervous system and restraining the abnormality of thinking process, in order to make patients remain the best psychological states and actively partner treatment and nursing. At the meantime, the somatic function and mental diseases of patients will be healed and treated faster. 


\section{The Psychological Feature AND HEALING REQUIREMENTS OF PATENTS WITH LIMB DYSFUNCTION}

Patients with limb dysfunction mainly include the following two kinds: One is the patients with limb activity disorder caused by organic diseases or injury of limbs such as arthritis, post operation of joint replacement, amputation and post operation of replantation of severed limb; the other is the patients with limb dysfunction caused by central nervous system disorders, such as patients with limb movement disturbance caused by cerebral palsy in children, patients with abnormal gait caused by polio (also called infantile paralysis) and patients who cannot be controlled by consciousness because of stroke. Except for the disability of limbs, the patients also suffer from psychological features of anxiety, loneliness, self-abasement, sensitivity and suspicion caused by the loss of somatic function and mental diseases. Patients with limb dysfunction fail to complete the regular movement because upper limbs or lower limbs lose movement function and control ability, so they have special requirements for the healing environment.

The requirements of patients with limb dysfunction for healing consist of the requirement for physical rehabilitation and the requirement for psychological rehabilitation. Regarding the requirement for physical rehabilitation, patients expect to recover the physical health condition as soon as possible via the exclusive rehabilitation scheme formulated by therapist according to different situations in the professional healing environment with good curative effects; in terms of the requirement for psychological rehabilitation, patients expect to receive treatment and nursing with the best mental states, eliminate anxiety and emotional stress and gradually build up initiative and self-confidence in recovering physical and psychological health in the healing environment with high safety and beautiful environment.

\section{DESIGN ELEMENTS OF THE HORTICULTURAL THERAPY IN THE HEALING ENVIRONMENT OF PATIENTS WITH LIMB DYSFUNCTION}

\section{A. Environment}

As the place for the implementation of horticultural therapy, environment should be different from common gardens and natural landscapes. It's necessary for designers to consider the place to implement the horticultural therapy, people and the medical implications contained in the place. The most important feature of the horticultural therapy is that patients can view the scenery in the garden in the environment surrounded by plants in the process of healing and treatment. They can directly feel the climatic changes of four seasons and the quiet growth of plants physically. Compared with killing time in the inpatient wards or waiting rooms without plants, its treatment and nursing effects will be enormously different. It can be seen that patients in the environment with various plants can exchange information with others, appreciate, touch and look after plants carefully and calm down slowly. In the environment, the patients' gender, age, disease and individual difference of ability are not barriers. Although they cannot behave so well as healthy person, they can obtain the affirmation of self-competence. Patients will be blissfully happy and gradually recover the normal relationship and friendly state with the society and the real life, accommodate to a new lifestyle and create a healing environment suitable for the patients with limb dysfunction.

\section{B. Plants}

As the main and basic materials of food materials, medicinal materials and construction materials, plants have been extensively applied. They are popular among people because they can cure diseases, recover health, give people vigor, relax our mind, relieve stress and improve the quality of life. The color, leaf shape, smell and stem leaf of plants will excite people's optic nerve, smell and antennal nerve and make hearing organs have diversified experience through the sound of wind, pitter-patter, the sound of water, chirp and birds' songs. The strong healthcare function of plants has been widely accepted by the mass. Some experts and scholars derive six methods of phototherapy, aromatherapy, herb therapy, flower arrangement therapy, bonsai therapy and forest therapy. Clinical tests prove that breathing in face of some specific plants will help patients to dredge the main and collateral channels, strengthen biochemical functions of organs and play a role of adjuvant therapy. For example, breathing in the ginkgo forest will benefit qi and constrain the lung, resolve dampness and cure diarrhea and have effective results for symptoms like chest distress and cough. The research on its mechanism shows: on one hand, many plants are able to release volatile substance that correspondingly adjusts human nerve and body fluid to influence people's physiology and psychology. For example, the volatile oil of many aromatic plants has pesticide effects and can be absorbed by human body to have good healing and treatment effects; on the other hand, the "photoelectric effect" and "point discharge" of plants can raise the concentration of negative air ions. Negative air ions have many rehabilitation and healthcare functions, adjust central nervous system and promote morphologic element of blood and the recovery of physical characteristics and ciliary movement on mucosa to effectively enhance the immunity of cells and body liquid of human. Besides, it has special effects of relieving tiredness and refection. Furthermore, the natural landscape formed by plants can tranquilize and allay excitement, relieve people's tension, ease impatience and relax our mind. Meanwhile, it successfully stimulates people's feelings of loving nature, cultivates our taste and purifies our soul. For example, lavender can relieve the symptoms of headache and insomnia. Geranium can relieve anxiety and fatigue. In a word, the healing environment created by designers has all-round influence on human body. The conscious selection and arrangement of plants in the healing environment will realize the healing of patients' body and soul. However, the slow effects and the absence of immediate effects like drugs also lead to the difficult implementation and popularization of the horticultural therapy.

\section{Patients}

The basic principle of design is people oriented. The pathogenesis, symptom, healing and treatment methods of different patients are different. Especially for the patients with limb dysfunction and balance dysfunction caused by balancing organs such as sensory nervous system, vestibular nerve 
system, epencephalon, spinal cord and basal nuclei or other central neuropathy, they need the careful observation of therapists in horticultural activities. Volunteers or nurses are allocated to help them. What's more, because the limbs are defective and the movement is slow, the patients with limb dysfunction feel "their techniques are not equal to others" and "they are inferior to others". Therefore, therapists should consider the complexity of horticultural operation and reduce meticulous contents involving horticultural operation and let these patients participate in the horticultural activities in their power. In this way, they will acquire certain sensory compensation at the meantime build up their body in the round Patients will develop coordination, improve sociability and get satisfaction and increase self-confidence. Besides, when improving skills, the patients gradually overcome inferiority complex and play a role in psychology compensating and achieve self-improvement and earn their own living.

\section{Methods AND STRATEgIES OF HORTICUltURAL} THERAPY IN THE HEALING ENVIRONMENT OF PATIENTS WITH LIMB DYSFUNCTION

\section{A. The Place Is Safe and Barrier Free}

Safety is the primary prerequisite to design the activity space of patients with limb dysfunction. The implementation of horticultural therapy for the patients with limb dysfunction in the healing environment must promote the interaction between patients and the natural world when the facilities and equipment are safe and convenient. In consideration of the safety of patients' activities, the places to implement the horticultural therapy must follow the principle that the location is safe, the facility is safe, the pavement is safe and the waterscape is safe. In terms of site location, the activity space of the patients with limb dysfunction should be far away from the major traffic aisles for motor vehicles and road intersections, transformer substation, garbage collection station, centralized parking lots and the entrance to basement parking, toxic substance, polluted water source and high noise pollution that are adverse to the life of patients. Places with breeze, sufficient sunlight and proper shading are priority. In addition, the places selected should be convenient for nurses to monitor and nurse; with regard to facilities, we should choose facilities and equipment with appropriate sizes and guarantee their sturdiness and durability. For example, the pant consoles to implement the horticultural therapy for patients should be easy to operate and firm. If the patient can only operate with one hand, the flower pot on the console should stay still. Flower scoops for transplantation are replaced by spoons. Slips are cut by plastic knives. Clip must use portable scissors. Proper operation and tools should be provided so that patients can complete the operation without the help of others; in terms of the pavement, materials that can protect the ground should be selected. Besides, they must be slip resistance and avoid the serious glare under the sunlight; in terms of the waterscape, the depth of water should not exceed 0.3 meters. The ground around the pool and the materials of barricade should be skid resistance. The bottom of the pool also needs anti-skid treatment. At the meantime, plants such as moss and algae must be cleared away to keep the water clean. The bridges across the river must be wide enough and have guard bars and handrails.

\section{B. Plant Furnishing Has Clear Target}

China has plentiful plant resources that have provided good conditions for the construction of healing environment. Generally speaking, plant furnishing enhances people's feelings through the color of plants, leaf shape, smell and the texture of stem leaf. For the patients with limb dysfunction that lose the ability of walking, from the perspective of vision, plants with cool tones easily create quiet environment and make irritable patients calm down, remove troubles and relax their minds. Large area of plants with cool tones can be arranged in the healing environment. On the other hand, plants with warm tones can build warm environment and inspire effete and upset patients with enthusiasm and induce excited emotions and its area should be properly controlled in the healing environment; from the perspective of smell, tress and shrubs such as sweet-scented osmanthus, banana shrub, clove, crape myrtle, Aglaia odorata, wintersweet, geranium, Arabian jasmine flower, cape jasmine flower and thyme can be provided. The potpourri of these plants can alleviate nerves and the mental stress of patients; from the perspective of touch, plants with potential safety hazard should be avoided, such as llex cornuta, pyracantha fortuneana and yucca gloriosa that have needle and thorns. It's necessary to reasonably make use of the different texture of plants and actively guide patients to participate in the activity of experiencing horticultural therapy to properly improve the capacity of patients' limbs for action, effectively alleviate diseases and promote rapid healing and treatment; from the perspective of gestation, patients can adjust mind and body through fruit and vegetable picking, eating and feeling the pleasure of harvest; from the perspective of auditory sense, plants must combine with other landscape elements. The sound of wind, pitter-patter, the sound of water and yell of animals are used to create audio landscapes such as "raindrops drummed rhythmically against the banana leaves", "listen to the sound of billows in bamboo forest" and "birds" twitter and fragrance of flowers" to achieve the expected results of pleasing and alleviating the mind and body of patients. All in all, the target of implementing the horticultural therapy must be clear. It mainly concerns the relationship between human and plants. That's to say, people cultivate plants and seek novelty of them. Observation and study have shown the horticultural therapy has seven functions namely the utility of production, economic utility, spiritual utility, environmental utility, social utility, educational utility and body utility. For the patients with limb dysfunction, the key point of the horticultural therapy is to train patients to obtain skills used in their daily life. The labor or operation of them in the process of horticultural therapy can help them recover holding function, improve occupational skills, strengthen social adaptation ability and achieve the desired effects of healing and treatment.

\section{Build Up Diversified Experience Space}

Rehabilitation hospitals use physical instruments or the repeat practice of fixed actions to train patients' limbs and sense organs. However, the horticultural therapy turns boring practice into the experiential activities to keep happy. For 
patients with limb dysfunction after amputation or without strong hand, learning control again through cultivating plants is more interesting than the implementation of mechanical physical exercises. Many repeated horticultural operations like watering, planting, clipping and weeding enable patients to enjoy happy activities while using assistive devices. The experience in the activity space of patients with limb dysfunction mainly includes action experience and feeling experience. The horticultural therapy mainly lets patients participate in the process of cultivating and looking after plants, waiting for their growth, blooming and bearing fruits. The process of cultivating plants and behaviors of harvesting vegetables, flowers and fruits make patients deeply perceive the law of life and then create the intimate relationship between human and plants. It even makes people's emotions depend on the growth of plants. When patients put in spirit and hope and look forward to the final harvest in the process of cultivating plants, plants have gone deep into the subconscious of patients. It helps patients to establish self-confidence, not only meeting people's instinctive desire of reaping the harvest and their desire for seeking novelty in the process of plants' growth but also realizing the exchange of human and nature in the experiential activities such as viewing, smelling, touching, listening, harvesting and tasting.

When it comes to the establishment of diversified experience space, first, the healing environment should have various space types for selection, including the private space suitable for staying alone, the public space suitable for contact, the wide open space to enjoy sunshine, the shade place away from the blazing sun, the viewing place to expand horizon and the micro-landscape; second, in the healing environment, we should pay attention to shaping sensory stimuli. Unique colors, sweet aroma, plentiful texture and the voice that conforms to life rhythm are used to stimulate patients' visual sense, smell, touch and auditory nerves, improve their sensitivity. Besides, the sound of wind, pitter-patter, and the sound of water and the yell of animals are introduced consciously and controlled within the proper volume range to achieve the desired effects of pleasing and alleviating the mind and body of patients.

\section{Establish the Perfect Treatment Plans and Management Methods of Horticultural Therapy}

It spends a relatively long time to make the horticultural therapy achieve the effects of healing and treatment. It's necessary to match professional and formulate exclusive treatment plans according to the characteristics of each patient's symptom. For example, for the patients whose upper and lower limbs cannot move because of dysfunction, therapists can discuss with him about the plants arranged around the bed every day, the modeling, color, style and match of plants, and let the "five senses" of plants stimulate patients' central nervous to reach treatment effects; for the patients who sit in wheelchair because of the dysfunction of lower limbs, framed flower bed that matches with the height of wheelchair can be provided for patients to conduct horticultural operation via sitting in the wheelchair; for the patients with one-sided limb dysfunction, the size, length, weight and height of tools are adjusted according to the physical condition of individuals so that the patients can complete all the horticultural operations with one hand; for the patients who have difficulty in action because of part limb dysfunction, regularly watering the plants needs the assistance of volunteers or nurses. In a word, regardless of the situation of limbs of the patients with limb dysfunction, the patients' remaining capacity for action are excavated as far as possible to coordinate with the healing and treatment brought about by the horticultural therapy. On the other hand, horticultural activities also can provide interesting ways for patients to improve their functions and regain the health of their limbs.

The daily management of horticultural therapy mainly refers to the management of everyday activity records, personal records, treatment plans, evaluation results and reports and the risk management of treatment objects to provide the site management of horticultural activities under the optimum state. It's worth noting that the risk management should base on the patients' barrier, grasp the patients' physical and mental state in the process of activity, and determine operation time, operation level and rest period to ensure that the patients will blissfully coordinate with the horticultural therapy; the site management means regularly managing the conditions of horticultural fields. In implementing the horticultural therapy, we must prepare the plants as materials in advance so that various horticultural operations will be conducted in different seasons.

\section{CONCLUSION}

It's unrealistic to heal the patients with limb dysfunction only through depending on the horticultural therapy. But it's feasible to formulate corresponding supplementary plans of the horticultural therapy for patients with limb dysfunction in the healing environment. Firstly, the healing environment belongs to a common social physical environment. The effects of implementing behavioral intervention in the gardening scenario are often superior to that of simply depending on drug therapy or carrying on mechanical training; secondly, the environmental factors such as the fresh air and suitable humidity in the healing environment can directly act on the patients' body and relieve their stress and make them feel relaxed. It's beneficial to the health of patients who are isolated in inpatient wards or waiting rooms for a long time; finally, as a way of training limbs, the horticultural therapy is the activity with strong interestingness and relatively low difficult. It's easy for patients to complete horticultural operation. The horticultural activities with proper intensity will gradually improve the motor function of patients. Besides, the healing and treatment of patients with limb dysfunction through the horticultural therapy should be highly valued by the society. Relevant personnel also should integrate the horticultural therapy and the healing environment to conduct deep research and practice, promote the exchange and cooperation between different professional fields, and create the rehabilitation space that is suitable for the patients with limb dysfunction at the same time promote the diversified development of designing healing environment. 


\section{REFERENCES}

[1] Li Shuhua. Introduction to Horticultural Therapy [M], Beijing: China Forestry Publishing House, 2011

[2] Li Shuhua. Origin and Development of Horticultural Therapy [J], Agricultural Science, Technology and Information: Modern Landscape Architecture, 2013(4): 1-2

[3] Ivarsson C T, Grahn P. Differently designed parts of a garden support different types of recreational walks: evaluating a healing garden by participatory observation [J].Landscape Research, 2012, 37 (5):519-537.

[4] (America)Dave Camp. Healing Garden [M], Guilin: Guangxi Normal University Press, 2016

[5] Guo Jing, Xu Zhao, He Rui. Analysis on the Design Methods of the Healing Space Suitable for the Patients with Limb Dysfunction [J], Furniture and Interior Design, 2016(11): 54-55 